Теорія Ймовір. та Матем. Статист. Вип. 75, 2006
Theor. Probability and Math. Statist.

No. 75, 2007, Pages 109-119 S 0094-9000(08)00718-7

Article electronically published on January 24, 2008

\title{
ON THE PROBLEM OF FILTRATION FOR VECTOR STATIONARY SEQUENCES
}

UDC 519.21

\author{
M. P. MOKLYACHUK AND O. YU. MASYUTKA
}

\begin{abstract}
We study the problem of optimal linear estimation of the functional $A \vec{\xi}=\sum_{j=0}^{\infty} \vec{a}(j) \vec{\xi}(-j)$ depending on unknown values of a vector stationary sequence $\vec{\xi}(j)=\left\{\xi_{k}(j)\right\}_{k=1}^{T}$ from observations upon the sequence $\vec{\xi}(j)+\vec{\eta}(j)$ for $j \leq 0$ where $\vec{\eta}(j)=\left\{\eta_{k}(j)\right\}_{k=1}^{T}$ is a vector stationary sequence, being uncorrelated with $\vec{\xi}(j)$. We obtain relations for the mean square error and spectral characteristic of the optimal estimator of the functional. We also find the least favorable spectral densities and minimax (robust) spectral characteristics of optimal estimators of the functional for a particular class $D$ of spectral densities.
\end{abstract}

\section{INTRODUCTION}

The main assumption of the classical theory of interpolation, extrapolation, and filtration of stationary stochastic processes is that the spectral densities of the processes are known. However, complete information about the spectral densities is not available in most of the practical cases. Parametric and nonparametric estimators of the spectral densities serve to solve this problem. There are some other (heuristic) methods to evaluate initial estimates of spectral densities. Having obtained initial estimates of the densities, the classical theory is applied under the assumption that the estimators are true densities. Particular examples show (see Vastola and Poor [1]) that this procedure may essentially increase the error. Thus it is worthwhile to look for estimators that are optimal for all densities belonging to a certain class of spectral densities. Estimators possessing this property are called minimax, since they minimize the maximal value of the error. A survey of results of the minimax method of estimation can be found in the paper by Kassam and Poor [2]. The methods of game theory are applied for the first time to the problems of extrapolation in the paper by Grenander 3. Franke 4, 5] studied the problem of extrapolation for stationary sequences with the help of methods of convex optimization.

The problems of extrapolation, interpolation, and filtration for stationary sequences and processes are studied in the papers by Moklyachuk [6] 8 . In this paper, we consider the problem of optimal linear estimation of the functional

$$
A \vec{\xi}=\sum_{j=0}^{\infty} \vec{a}(j) \vec{\xi}(-j)
$$

2000 Mathematics Subject Classification. Primary 60G35; Secondary 62M20, 93E10, 93E11.

Key words and phrases. Vector stationary sequence, observations in the presence of noise, optimal linear estimator, mean square error, spectral characteristic, least favorable spectral density, minimax (robust) spectral characteristic. 
depending on unknown values of a vector stationary sequence $\vec{\xi}(j)=\left\{\xi_{k}(j)\right\}_{k=1}^{T}$ whose spectral density is $F(\lambda)$. We estimate the functional from observations upon the sequence $\vec{\xi}(j)+\vec{\eta}(j)$ for $j \leq 0$, where $\vec{\eta}(j)=\left\{\eta_{k}(j)\right\}_{k=1}^{T}$ is a vector stationary sequence that is uncorrelated with $\vec{\xi}(j)$ and has spectral density $G(\lambda)$.

\section{The Classical Method of FiLtration}

Assume that the coefficients $\vec{a}(j)=\left\{a_{k}(j)\right\}_{k=1}^{T}$ that determine the functional $A \vec{\xi}$ are such that

$$
\sum_{j=0}^{\infty} \sum_{k=1}^{T}\left|a_{k}(j)\right|<\infty, \quad \sum_{j=0}^{\infty}(j+1)\|\vec{a}(j)\|^{2}<\infty,
$$

where

$$
\|\vec{a}(j)\|^{2}=\sum_{k=1}^{T}\left|a_{k}(j)\right|^{2} .
$$

Then the second moment of the functional $A \vec{\xi}$ is finite and the operator $A$ defined below is compact. The stationary sequence $\vec{\xi}(j)+\vec{\eta}(j)$ admits an expansion in the form of the canonical moving average

$$
\vec{\xi}(j)+\vec{\eta}(j)=\sum_{u=-\infty}^{j} d(j-u) \vec{\varepsilon}(u)
$$

if the matrix of spectral densities

$$
F(\lambda)+G(\lambda)=\left\{f_{i j}(\lambda)+g_{i j}(\lambda)\right\}_{i, j=1}^{T}
$$

of the sequence $\vec{\xi}(j)+\vec{\eta}(j)$ admits the canonical factorization [9]

$$
F(\lambda)+G(\lambda)=d\left(e^{i \lambda}\right) d^{*}\left(e^{i \lambda}\right), \quad d\left(e^{i \lambda}\right)=\sum_{k=0}^{\infty} d(k) e^{-i k \lambda},
$$

where $d(k)=\left\{d_{i j}(k)\right\}_{i=1, \ldots, T}^{j=1, \ldots, m}$ and $\vec{\varepsilon}(u)=\left\{\varepsilon_{k}(u)\right\}_{k=1}^{m}$ is a vector stationary white noise sequence such that $\mathrm{E}\left|\varepsilon_{k}(u)\right|^{2}=1, k=1, \ldots, m$, and $\mathrm{E} \varepsilon_{i}(t) \overline{\varepsilon_{j}(s)}=0, t \neq s$. If at least one of the densities $F(\lambda)$ or $G(\lambda)$ is regular, then the spectral density $F(\lambda)+G(\lambda)$ admits a factorization. Regular spectral densities $F(\lambda)$ and $G(\lambda)$ admit the canonical factorization

$$
\begin{array}{ll}
F(\lambda)=\varphi\left(e^{i \lambda}\right) \varphi^{*}\left(e^{i \lambda}\right), & \varphi\left(e^{i \lambda}\right)=\sum_{k=0}^{\infty} \varphi(k) e^{-i k \lambda} \\
G(\lambda)=\psi\left(e^{i \lambda}\right) \psi^{*}\left(e^{i \lambda}\right), & \psi\left(e^{i \lambda}\right)=\sum_{k=0}^{\infty} \psi(k) e^{-i k \lambda}
\end{array}
$$

where $\varphi(k)=\left\{\varphi_{i j}(k)\right\}_{i=1, \ldots, T}^{j=1, \ldots, m}$ and $\psi(k)=\left\{\psi_{i j}(k)\right\}_{i=1, \ldots, T}^{j=1, \ldots, m}$.

Let

$$
\hat{A} \vec{\xi}=\int_{-\pi}^{\pi} h\left(e^{i \lambda}\right)\left(Z^{\xi}(d \lambda)+Z^{\eta}(d \lambda)\right)=\int_{-\pi}^{\pi} \sum_{k=1}^{T} h_{k}\left(e^{i \lambda}\right)\left(Z_{k}^{\xi}(d \lambda)+Z_{k}^{\eta}(d \lambda)\right)
$$

be the mean square error of the linear estimator of the functional $A \vec{\xi}$, where

$$
Z^{\xi}(\Delta)=\left\{Z_{k}^{\xi}(\Delta)\right\}_{k=1}^{T} \quad \text { and } \quad Z^{\eta}(\Delta)=\left\{Z_{k}^{\eta}(\Delta)\right\}_{k=1}^{T}
$$


are orthogonal random measures of the sequences $\vec{\xi}(j)$ and $\vec{\eta}(j)$, respectively, and

$$
h\left(e^{i \lambda}\right)=\sum_{k=0}^{\infty} \vec{h}(k) e^{-i k \lambda}
$$

is the spectral characteristic of the estimator. The mean square error can be evaluated by

$$
\begin{aligned}
\Delta(h ; F, G)= & \mathrm{E}|A \vec{\xi}-\hat{A} \vec{\xi}|^{2} \\
= & \frac{1}{2 \pi} \int_{-\pi}^{\pi}\left[A\left(e^{i \lambda}\right) G(\lambda) A^{*}\left(e^{i \lambda}\right)\right. \\
& +\left(A\left(e^{i \lambda}\right)-h\left(e^{i \lambda}\right)\right)(F(\lambda)+G(\lambda))\left(A\left(e^{i \lambda}\right)-h\left(e^{i \lambda}\right)\right)^{*} \\
& -\left(A\left(e^{i \lambda}\right)-h\left(e^{i \lambda}\right)\right) G(\lambda) A^{*}\left(e^{i \lambda}\right) \\
& \left.-A\left(e^{i \lambda}\right) G(\lambda)\left(A\left(e^{i \lambda}\right)-h\left(e^{i \lambda}\right)\right)^{*}\right] d \lambda \\
& +\sum_{k=0}^{\infty} \sum_{j=0}^{\infty} \sum_{p=-\infty}^{\infty} \sum_{p=-\infty}^{\min (k, j)} \vec{a}(k) \psi(k-p) \psi^{*}(j-p) \vec{a}^{*}(j) \\
& -\sum_{k=0}^{\infty} \sum_{j=0}^{\infty} \sum_{p=-\infty}^{\min (k, j)}(\vec{a}(k)-\vec{h}(k)) \psi(k-p) \psi^{*}(j-p) \vec{a}^{*}(j) \\
& -\sum_{k=0}^{\infty} \sum_{j=0}^{\infty} \sum_{p=-\infty}^{\min (k, j)} \vec{a}(k) \psi(k-p) d^{*}(j-p)(\vec{a}(j)-\vec{h}(j))^{*} \\
= & \|\Psi a\|^{2}+\|D(a-h)\|^{2}-\langle\Psi(j(a-h), \Psi a\rangle-\langle\Psi a, \Psi(a-h)\rangle,
\end{aligned}
$$

where

$$
\begin{gathered}
A\left(e^{i \lambda}\right)=\sum_{j=0}^{\infty} \vec{a}(j) e^{-i j \lambda}, \quad\|\Psi a\|^{2}=\sum_{k=0}^{\infty}\left\|(\Psi a)_{k}\right\|^{2}, \quad(\Psi a)_{k}=\sum_{l=0}^{k} \vec{a}(l) \psi(k-l), \\
\|D(a-h)\|^{2}=\sum_{k=0}^{\infty}\left\|(D(a-h))_{k}\right\|^{2}, \quad(D(a-h))_{k}=\sum_{l=0}^{k}(\vec{a}(l)-\vec{h}(l)) d(k-l), \\
\langle\Psi(a-h), \Psi a\rangle=\overline{\langle\Psi a, \Psi(a-h)\rangle}=\sum_{k=0}^{\infty}\left\langle(\Psi(a-h))_{k},(\Psi a)_{k}\right\rangle .
\end{gathered}
$$

The spectral characteristic $h(F, G)$ of the optimal linear estimator of the functional $A \vec{\xi}$ for given spectral densities $F(\lambda)$ and $G(\lambda)$ is determined by the condition

$$
\Delta(F, G)=\Delta(h(F, G) ; F, G)=\min _{h \in L_{2}^{-}(F+G)} \Delta(h ; F, G)
$$

where $L_{2}^{-}(F+G)$ is the subspace of the space $L_{2}(F+G)$ generated by functions of the form $e^{i n \lambda} \delta_{k}, \delta_{k}=\left\{\delta_{k l}\right\}_{l=1}^{T}, k=1, \ldots, T, n \leq 0$. Here $\delta_{k k}=1$ and $\delta_{k l}=0$ for $k \neq l$. If the spectral densities admit canonical representations (3) and (5), then the mean square error of the optimal linear estimator $\hat{A} \vec{\xi}$ equals

$$
\Delta(F, G)=\|\Psi a\|^{2}-\left\|B^{*} \Psi^{*} \Psi a\right\|^{2}=\left\langle\mathbf{c}_{G}, a\right\rangle-\left\|\mathbf{C}_{G} b^{*}\right\|^{2},
$$


where

$$
\begin{gathered}
\mathbf{c}_{G}(k)=\left(\Psi^{*} \Psi a\right)_{k}=\sum_{l=0}^{\infty}(\Psi a)_{l+k} \psi^{*}(l) \\
=\sum_{l=0}^{k-1} \vec{a}(l) \sum_{m=0}^{\infty} \psi(m+k-l) \psi^{*}(m)+\sum_{l=0}^{\infty} \vec{a}(k+l) \sum_{m=0}^{\infty} \psi(m) \psi^{*}(m+l), \\
\left(B^{*} \Psi^{*} \Psi a\right)_{k}=\sum_{l=0}^{\infty}\left(\Psi^{*} \Psi a\right)_{l+k} b^{*}(l), \\
\left(\mathbf{C}_{G} b^{*}\right)_{k}=\sum_{l=0}^{\infty} c_{G}(l+k) b^{*}(l),
\end{gathered}
$$

and

$$
b\left(e^{i \lambda}\right)=\left\{b_{i j}\left(e^{i \lambda}\right)\right\}_{i=1, \ldots, m}^{j=1, \ldots, T}
$$

is a matrix function such that $b\left(e^{i \lambda}\right)=\sum_{k=0}^{\infty} b(k) e^{-i k \lambda}$ and $b\left(e^{i \lambda}\right) d\left(e^{i \lambda}\right)=I_{m}$, where $I_{m}$ is the unit $m \times m$ matrix. The spectral characteristic $h(F, G)$ of the optimal estimator is given by

$$
h(F, G)=A\left(e^{i \lambda}\right)-r_{G}\left(e^{i \lambda}\right) b\left(e^{i \lambda}\right), \quad r_{G}\left(e^{i \lambda}\right)=\sum_{k=0}^{\infty}\left(\mathbf{C}_{G} b^{*}\right)_{k} e^{-i k \lambda} .
$$

If the spectral densities admit canonical representations (3) and (4), then the mean square error and spectral characteristic of the optimal estimator can be evaluated as follows:

$$
\begin{gathered}
\Delta(F, G)=\left\langle\mathbf{c}_{F}, a\right\rangle-\left\|\mathbf{C}_{F} b^{*}\right\|^{2} \\
h(F, G)=r_{F}\left(e^{i \lambda}\right) b\left(e^{i \lambda}\right), \quad r_{F}\left(e^{i \lambda}\right)=\sum_{k=0}^{\infty}\left(\mathbf{C}_{F} b^{*}\right)_{k} e^{-i k \lambda},
\end{gathered}
$$

where

$$
\mathbf{c}_{F}(k)=\left(\Phi^{*} \Phi a\right)_{k}=\sum_{l=0}^{\infty}(\Phi a)_{l+k} \varphi^{*}(l), \quad(\Phi a)_{k}=\sum_{l=0}^{k} \vec{a}(l) \varphi(k-l) .
$$

We have

$$
\Delta(F, G)=\sigma^{2} \sum_{k=0}^{\infty}\|\vec{a}(k)\|^{2}-\sigma^{4}\left\|A b^{*}\right\|^{2}
$$

in the case where $\vec{\eta}(j)$ or $\vec{\xi}(j)$ is a sequence of coordinatewise uncorrelated random vectors with the variance $\sigma^{2}$ (a vector white noise sequence). We put

$$
\left\|A b^{*}\right\|^{2}=\sum_{k=0}^{\infty}\left\|\left(A b^{*}\right)_{k}\right\|^{2}, \quad\left(A b^{*}\right)_{k}=\sum_{l=0}^{\infty} \vec{a}(k+l) b^{*}(l)
$$

in equality (11).

The mean square error of the optimal linear estimator of $\vec{a}(N) \vec{\xi}(-N)$ constructed from observations $\vec{\xi}(j)+\vec{\eta}(j)$ for $j \leq 0$ can be evaluated in this case as follows:

$$
\Delta(F, G)=\sigma^{2} \sum_{k=0}^{T}\left|a_{k}(N)\right|^{2}-\sigma^{4} \sum_{k=0}^{N}\left\|\vec{a}(N) b^{*}(k)\right\|^{2} .
$$

The coefficients $b(k)$ can be found from the factorization (3) of the density $F(\lambda)+\sigma^{2} I_{T}$.

Therefore the following result holds. 
Theorem 2.1. Let $\Delta(F, G)$ be the mean square error of the optimal linear estimator of the functional $A \vec{\xi}$ depending on unknown values of a vector sequence $\vec{\xi}(j)$ and constructed from observations upon the sequence $\vec{\xi}(j)+\vec{\eta}(j)$ for $j \leq 0$, where $\vec{\xi}(j)$ and $\vec{\eta}(j)$ are uncorrelated vector stationary sequences whose spectral densities, $F(\lambda)$ and $G(\lambda)$, respectively, admit canonical representations (3), (5) or (3), (4). Then $\Delta(F, G)$ is given by relations (7), (9). The spectral characteristic $h(F, G)$ of the optimal filtration is given by relations (8), (10).

Remark 2.1. If a vector stationary sequence is of a maximal rank (that is, if $m=T$ ), then the matrix function $b\left(e^{i \lambda}\right)$ is the inverse matrix to $d\left(e^{i \lambda}\right)$, that is, $b\left(e^{i \lambda}\right)=d^{-1}\left(e^{i \lambda}\right)$. If a vector stationary sequence is of rank 1 (that is, if $m=1$ ), then the matrix function $b\left(e^{i \lambda}\right)$ is a vector row $b\left(e^{i \lambda}\right)=\left\{b_{k}\left(e^{i \lambda}\right)\right\}_{k=1}^{T}$ that can be evaluated from the equation $\sum_{k=1}^{T} b_{k}\left(e^{i \lambda}\right) d_{k}\left(e^{i \lambda}\right)=1$.

Example 1. Consider the problem of estimation of a random variable

$$
\vec{a}(0) \vec{\xi}(0)=a_{1}^{0} \xi_{1}(0)+a_{2}^{0} \xi_{2}(0)
$$

from observations of the sequence $\vec{\xi}(j)+\vec{\eta}(j), j \leq 0$, in the case where

$$
\begin{gathered}
F(\lambda)=\left(\begin{array}{cc}
f(\lambda) & f(\lambda) \\
f(\lambda) & f(\lambda)+f_{1}(\lambda)
\end{array}\right), \quad f(\lambda)=\frac{P_{1}^{2}}{\mid 1-b_{1} e^{-\left.i \lambda\right|^{2}}}, \quad f_{1}(\lambda)=\frac{P_{2}^{2}}{\left|1-b_{2} e^{-i \lambda}\right|^{2}}, \\
G(\lambda)=\left(\begin{array}{cc}
g(\lambda) & g(\lambda) \\
g(\lambda) & g(\lambda)+g_{1}(\lambda)
\end{array}\right), \quad g(\lambda)=\sigma^{2}, \quad g_{1}(\lambda)=\frac{P_{3}^{2}}{\left|1-b_{3} e^{-i \lambda}\right|^{2}} .
\end{gathered}
$$

Then $F(\lambda)+G(\lambda)=d\left(e^{i \lambda}\right) d^{*}\left(e^{i \lambda}\right)$, where

$$
\begin{gathered}
d\left(e^{i \lambda}\right)=\left(\begin{array}{cc}
A \frac{1-\beta e^{-i \lambda}}{1-b_{1} e^{-i \lambda}} & 0 \\
A \frac{1-\beta e^{-i \lambda}}{1-b_{1} e^{-i \lambda}} & B \frac{1-\gamma e^{-i \lambda}}{\left(1-b_{2} e^{-i \lambda}\right)\left(1-b_{3} e^{-i \lambda}\right)}
\end{array}\right), \quad A^{2}=\frac{\sigma^{2} b_{1}}{\beta}, \quad B^{2}=\frac{P_{2}^{2} b_{3}+P_{3}^{2} b_{2}}{\gamma}, \\
\beta=\frac{1}{2}\left(b_{1}+b_{1}^{-1}\right)(1+\theta)-\left[\frac{1}{4}(1+\theta)^{2}\left(b_{1}+b_{1}^{-1}\right)^{2}-1\right]^{1 / 2}, \\
\theta=\frac{P_{1}^{2}}{\sigma^{2}\left(1+b_{1}^{2}\right)},
\end{gathered}
$$

and $\gamma$ is a root (whose absolute value is less than 1) of the equation

$$
z^{2}-\frac{P_{2}^{2}+P_{3}^{2}+P_{2}^{2} b_{3}^{2}+P_{3}^{2} b_{2}^{2}}{P_{2}^{2} b_{3}+P_{3}^{2} b_{2}} z+1=0
$$

Let us evaluate the inverse matrix:

$$
b\left(e^{i \lambda}\right)=d^{-1}\left(e^{i \lambda}\right)=\left(\begin{array}{cc}
\frac{1}{A} \frac{1-b_{1} e^{-i \lambda}}{1-\beta e^{-i \lambda}} & 0 \\
-\frac{1}{B} \frac{\left(1-b_{2} e^{-i \lambda}\right)\left(1-b_{3} e^{-i \lambda}\right)}{1-\gamma e^{-i \lambda}} & \frac{1}{B} \frac{\left(1-b_{2} e^{-i \lambda}\right)\left(1-b_{3} e^{-i \lambda}\right)}{1-\gamma e^{-i \lambda}}
\end{array}\right)
$$

and the spectral characteristic of the optimal estimator

$$
h\left(e^{i \lambda}\right)=\left(h_{1}\left(e^{i \lambda}\right), h_{2}\left(e^{i \lambda}\right)\right)=r_{F}\left(e^{i \lambda}\right) b\left(e^{i \lambda}\right),
$$

where

$$
r_{F}\left(e^{i \lambda}\right)=\sum_{k=0}^{\infty}\left(\mathbf{C}_{F} b^{*}\right)_{k} e^{-i k \lambda}, \quad\left(\mathbf{C}_{F} b^{*}\right)_{k}=\sum_{l=0}^{\infty} \mathbf{c}_{F}(l+k) b^{*}(l) .
$$


Since $\mathbf{c}_{F}(l)=\vec{a}(0) \sum_{k=0}^{\infty} \varphi(k+l) \varphi^{*}(k)=\left(c_{1}(l), c_{2}(l)\right)$, we have

$$
\begin{aligned}
h_{1}\left(e^{i \lambda}\right)= & \frac{\left(a_{1}^{0}+a_{2}^{0}\right) P_{1}^{2}}{A^{2}} \frac{1-b_{1} e^{-i \lambda}}{1-\beta e^{-i \lambda}}\left[\frac{1-b_{1} e^{i \lambda}}{1-\beta e^{i \lambda}} \cdot \frac{1}{\left|1-b_{1} e^{-i \lambda}\right|^{2}}\right]_{-} \\
& -\frac{a_{2}^{0} P_{2}^{2}}{B^{2}} \frac{\left(1-b_{2} e^{-i \lambda}\right)\left(1-b_{3} e^{-i \lambda}\right)}{1-\gamma e^{-i \lambda}}\left[\frac{\left(1-b_{2} e^{i \lambda}\right)\left(1-b_{3} e^{i \lambda}\right)}{1-\gamma e^{i \lambda}} \frac{1}{\left|1-b_{2} e^{-i \lambda}\right|^{2}}\right]_{-},
\end{aligned}
$$

where

$$
c_{1}(l)=\left[\frac{\left(a_{1}^{0}+a_{2}^{0}\right) P_{1}^{2}}{\left|1-b_{1} e^{-i \lambda}\right|^{2}}\right]_{l}, \quad c_{2}(l)=c_{1}(l)+\left[\frac{a_{2}^{0} P_{2}^{2}}{\left|1-b_{2} e^{-i \lambda}\right|^{2}}\right]_{l}
$$

are the coefficients of $e^{-i l \lambda}$ in the Fourier expansions of the function $r_{F}$ and where $[f(\lambda)]_{-}$ stands for the Fourier transform of $f$ in negative powers $e^{-i k \lambda}, k \geq 0$. Considering

$$
\left[e^{i \mu \lambda} \frac{1}{\left(1-\gamma e^{i \lambda}\right)\left(1-b_{2} e^{-i \lambda}\right)}\right]_{-}=\frac{b_{2}^{\mu}}{1-\gamma b_{2}} \cdot \frac{1}{1-b_{2} e^{-i \lambda}}
$$

we obtain

$$
h_{1}\left(e^{i \lambda}\right)=\frac{a_{1}^{0}+a_{2}^{0}}{A^{2}\left(1-b_{1} \beta\right)} \frac{P_{1}^{2}}{1-\beta e^{-i \lambda}}-\frac{a_{2}^{0}\left(1-b_{2} b_{3}\right) P_{2}^{2}}{B^{2}\left(1-\gamma b_{2}\right)} \frac{1-b_{3} e^{-i \lambda}}{1-\gamma e^{-i \lambda}} .
$$

Similarly,

$$
h_{2}\left(e^{i \lambda}\right)=\frac{a_{2}^{0}\left(1-b_{2} b_{3}\right) P_{2}^{2}}{B^{2}\left(1-\gamma b_{2}\right)} \frac{1-b_{3} e^{-i \lambda}}{1-\gamma e^{-i \lambda}} .
$$

The mean square error is evaluated as follows:

$$
\Delta(F, G)=\frac{\left(a_{1}^{0}+a_{2}^{0}\right)^{2} P_{1}^{2}}{1-b_{1}^{2}}+\frac{\left(a_{2}^{0}\right)^{2} P_{2}^{2}}{1-b_{2}^{2}}-\frac{C^{2}}{1-b_{1}^{2}}-\frac{D^{2}}{1-b_{2}^{2}},
$$

where

$$
\begin{gathered}
C=\frac{\left(a_{1}^{0}+a_{2}^{0}\right) P_{1}^{2}}{A\left(1-b_{1} \beta\right)\left(1-b_{1}^{2}\right)}\left(1-\frac{b_{1}}{\beta}\right), \\
D=\frac{a_{2}^{0} P_{2}^{2}}{B\left(1-b_{2}^{2}\right)\left(1-b_{2} \gamma\right)}\left(1-\frac{b_{2}+b_{3}}{\gamma}+\frac{b_{2} b_{3}}{\gamma^{2}}\right) .
\end{gathered}
$$

Using the above results one can calculate the spectral characteristic

$$
h\left(e^{i \lambda}\right)=\left(h_{1}\left(e^{i \lambda}\right), h_{2}\left(e^{i \lambda}\right)\right)
$$

of the optimal estimator of the random variable $\vec{a}(N) \vec{\xi}(-N)$ :

$$
h_{1}\left(e^{i \lambda}\right)=\frac{\left(a_{1}^{N}+a_{2}^{N}\right) P_{1}^{2}}{A^{2}} p\left(e^{i \lambda}\right)-\frac{a_{2}^{N} P_{2}^{2}}{B^{2}} q\left(e^{i \lambda}\right), \quad h_{2}\left(e^{i \lambda}\right)=\frac{a_{2}^{N} P_{2}^{2}}{B^{2}} q\left(e^{i \lambda}\right),
$$

where

$$
\begin{gathered}
p\left(e^{i \lambda}\right)=\left(\frac{\beta e^{i \lambda}\left(1-\beta^{N} e^{i N \lambda}\right)}{1-\beta e^{i \lambda}}+\frac{1}{1-b_{1} e^{-i \lambda}}\right) \frac{e^{-i N \lambda}\left(1-b_{1} e^{-i \lambda}\right)}{\left(1-b_{1} \beta\right)\left(1-\beta e^{-i \lambda}\right)} \\
q\left(e^{i \lambda}\right)=\frac{1-b_{2} e^{-i \lambda}}{1-\gamma b_{2}} e^{-i N \lambda} \frac{1-b_{3} e^{-i \lambda}}{1-\gamma e^{-i \lambda}}\left(\frac{1-b_{3} b_{2}}{1-b_{2} e^{-i \lambda}}+\frac{\gamma e^{i \lambda}\left(1-\gamma^{N} e^{i N \lambda}\right)}{1-\gamma e^{i \lambda}}\left(1-\frac{b_{3}}{\gamma}\right)\right) .
\end{gathered}
$$

The mean square error is calculated analogously. 


\section{Minimax method of Linear Filtration}

One can use relations (1)-(11) if the matrices of spectral densities $F(\lambda)$ and $G(\lambda)$ of vector stationary sequences $\{\vec{\xi}(j)\}$ and $\{\vec{\eta}(j)\}$ are known. Otherwise, we apply the minimax approach to problems of estimation of functionals of vector stationary sequences assuming that a set $D=D_{F} \times D_{G}$ containing these densities is known.

Definition 3.1. Spectral densities $F^{0}(\lambda)$ and $G^{0}(\lambda)$ are called the least favorable in a class $D$ for the optimal linear filtration of the functional $A \vec{\xi}$ if

$$
\Delta\left(h\left(F^{0}, G^{0}\right) ; F^{0}, G^{0}\right)=\max _{(F, G) \in D} \Delta(h(F, G) ; F, G) .
$$

Examining relations (1)-(11) one can prove the following propositions.

Theorem 3.1. Spectral densities $F^{0}(\lambda)$ and $G^{0}(\lambda)$ admitting canonical factorizations (3)-(5) are the least favorable in a class $D$ for the optimal filtration of the functional $A \vec{\xi}$ if the coefficients of the factorization (3)-(5) are a solution of the following conditional extremum problem:

$$
\begin{gathered}
\Delta(F, G)=\left\langle\mathbf{c}_{G}, a\right\rangle-\left\|\mathbf{C}_{G} b^{*}\right\|^{2} \rightarrow \sup \\
G(\lambda)=\left(\sum_{k=0}^{\infty} \psi(k) e^{-i k \lambda}\right) \cdot\left(\sum_{k=0}^{\infty} \psi(k) e^{-i k \lambda}\right)^{*} \in D_{G} \\
F(\lambda)=\left(\sum_{k=0}^{\infty} d(k) e^{-i k \lambda}\right)\left(\sum_{k=0}^{\infty} d(k) e^{-i k \lambda}\right)^{*}-\left(\sum_{k=0}^{\infty} \psi(k) e^{-i k \lambda}\right)\left(\sum_{k=0}^{\infty} \psi(k) e^{-i k \lambda}\right)^{*} \in D_{F}
\end{gathered}
$$

or of the following conditional extremum problem:

$$
\begin{gathered}
\Delta(F, G)=\left\langle\mathbf{c}_{F}, a\right\rangle-\left\|\mathbf{C}_{F} b^{*}\right\|^{2} \rightarrow \sup \\
F(\lambda)=\left(\sum_{k=0}^{\infty} \varphi(k) e^{-i k \lambda}\right) \cdot\left(\sum_{k=0}^{\infty} \varphi(k) e^{-i k \lambda}\right)^{*} \in D_{F}, \\
G(\lambda)=\left(\sum_{k=0}^{\infty} d(k) e^{-i k \lambda}\right)\left(\sum_{k=0}^{\infty} d(k) e^{-i k \lambda}\right)^{*}-\left(\sum_{k=0}^{\infty} \varphi(k) e^{-i k \lambda}\right)\left(\sum_{k=0}^{\infty} \varphi(k) e^{-i k \lambda}\right)^{*} \in D_{G} .
\end{gathered}
$$

If one of the densities is known, then (12) and (13) are extremum problems with respect to the variable $b=\{b(k): k=0,1, \ldots\}$.

Theorem 3.2. Let the spectral density $G(\lambda)$ be known and regular. A spectral density $F^{0}(\lambda)$ admitting canonical representations (3), (4) is the least favorable in a class $D_{F}$ for the optimal linear filtration of the functional $A \vec{\xi}$ if

$$
F^{0}(\lambda)+G(\lambda)=\left(\sum_{k=0}^{\infty} d^{0}(k) e^{-i k \lambda}\right) \cdot\left(\sum_{k=0}^{\infty} d^{0}(k) e^{-i k \lambda}\right)^{*},
$$

where $d^{0}=\left\{d^{0}(k): k=0,1, \ldots\right\}$ are determined by the coefficients of the expansion of the matrix function $b\left(e^{i \lambda}\right): b\left(e^{i \lambda}\right) d\left(e^{i \lambda}\right)=I_{m}, b\left(e^{i \lambda}\right)=\sum_{k=0}^{\infty} b^{0}(k) e^{-i k \lambda}$, where

$$
b^{0}=\left\{b^{0}(k): k=0,1, \ldots\right\}
$$

is a solution of the following conditional extremum problem:

$$
F(\lambda)=\left(\sum_{k=0}^{\infty} d(k) e^{-i k \lambda}\right) \cdot\left(\sum_{k=0}^{\infty} d(k) e^{-i k \lambda}\right)^{*}-G(\lambda) \in D_{F} .
$$


Theorem 3.3. Let the spectral density $F(\lambda)$ be known and regular. A spectral density $G^{0}(\lambda)$ admitting canonical factorizations (3), (5) is the least favorable in a class $D_{G}$ for the optimal linear filtration of the functional $A \vec{\xi}$ if

$$
F(\lambda)+G^{0}(\lambda)=\left(\sum_{k=0}^{\infty} d^{0}(k) e^{-i k \lambda}\right) \cdot\left(\sum_{k=0}^{\infty} d^{0}(k) e^{-i k \lambda}\right)^{*},
$$

where $d^{0}=\left\{d^{0}(k): k \geq 0\right\}$ are determined by the coefficients $b^{0}=\left\{b^{0}(k): k \geq 0\right\}$ that are solutions of the following conditional extremum problem:

$$
\begin{gathered}
\left\|\mathbf{C}_{F} b^{*}\right\|^{2} \rightarrow \inf , \\
G(\lambda)=\left(\sum_{k=0}^{\infty} d(k) e^{-i k \lambda}\right) \cdot\left(\sum_{k=0}^{\infty} d(k) e^{-i k \lambda}\right)^{*}-F(\lambda) \in D_{G} .
\end{gathered}
$$

Definition 3.2. The spectral characteristic $h^{0}(\lambda)$ of the optimal linear estimator of the functional $A \vec{\xi}$ is called minimax (robust) if

$$
h^{0}(\lambda) \in H_{D}=\bigcap_{(F, G) \in D} L_{2}^{-}(F+G), \quad \min _{h \in H_{D}} \sup _{(F, G) \in D} \Delta(h ; F, G)=\sup _{(F, G) \in D} \Delta\left(h^{0} ; F, G\right) .
$$

The least favorable spectral densities $F^{0}(\lambda)$ and $G^{0}(\lambda)$ and minimax (robust) spectral characteristic $h^{0}(\lambda) \in H_{D}$ form a saddle point of the function $\Delta(h ; F, G)$. The saddle point inequalities

$$
\Delta\left(h ; F^{0}, G^{0}\right) \geq \Delta\left(h^{0} ; F^{0}, G^{0}\right) \geq \Delta\left(h^{0} ; F, G\right)
$$

hold for all $(F, G) \in D$ and for all $h \in H_{D}$ if $h^{0}=h\left(F^{0}, G^{0}\right) \in H_{D}$, where $\left(F^{0}, G^{0}\right)$ is a solution of the conditional extremum problem

$$
\Delta\left(h\left(F^{0}, G^{0}\right) ; F^{0}, G^{0}\right)=\sup _{(F, G) \in D} \Delta\left(h\left(F^{0}, G^{0}\right) ; F, G\right)
$$

with the following objective function:

$$
\begin{aligned}
\Delta\left(h\left(F^{0}, G^{0}\right) ; F, G\right)= & \frac{1}{2 \pi} \int_{-\pi}^{\pi} r_{G}\left(e^{i \lambda}\right) b^{0}\left(e^{i \lambda}\right) F(\lambda)\left(b^{0}\left(e^{i \lambda}\right)\right)^{*}\left(r_{G}\left(e^{i \lambda}\right)\right)^{*} d \lambda \\
& +\frac{1}{2 \pi} \int_{-\pi}^{\pi} r_{F}\left(e^{i \lambda}\right) b^{0}\left(e^{i \lambda}\right) G(\lambda)\left(b^{0}\left(e^{i \lambda}\right)\right)^{*}\left(r_{F}\left(e^{i \lambda}\right)\right)^{*} d \lambda
\end{aligned}
$$

and where the functions $r_{F}\left(e^{i \lambda}\right)$ and $r_{G}\left(e^{i \lambda}\right)$ are obtained from relations (8) and (10), respectively, considered for

$$
F(\lambda)=F^{0}(\lambda), \quad G(\lambda)=G^{0}(\lambda) .
$$

\section{The least favorable spectral Densities in the Class $D_{0,0}$}

Consider the problem of the minimax estimation of the functional $A \vec{\xi}$ of a vector stationary sequence $\vec{\xi}(j)$ for the following set of spectral densities:

$$
D_{0,0}=\left\{(F(\lambda), G(\lambda)): \frac{1}{2 \pi} \int_{-\pi}^{\pi} F(\lambda) d \lambda=P_{1}, \frac{1}{2 \pi} \int_{-\pi}^{\pi} G(\lambda) d \lambda=P_{2}\right\} .
$$

Following the Lagrange multipliers method we obtain the following equalities for the evaluation of the least favorable densities $F^{0}(\lambda)$ and $G^{0}(\lambda)$ :

$$
\begin{aligned}
& r_{G}\left(e^{i \lambda}\right) b^{0}\left(e^{i \lambda}\right)=\vec{\alpha}, \\
& r_{F}\left(e^{i \lambda}\right) b^{0}\left(e^{i \lambda}\right)=\vec{\beta},
\end{aligned}
$$


where $\vec{\alpha}=\left(\alpha_{1}, \ldots, \alpha_{T}\right)^{\top}$ and $\vec{\beta}=\left(\beta_{1}, \ldots, \beta_{T}\right)^{\top}$ are undefined Lagrange multipliers. This implies that the least favorable spectral densities are

$$
\begin{aligned}
& F^{0}(\lambda)+G^{0}(\lambda)=\vec{\gamma}\left(\sum_{k=0}^{\infty}\left(C_{G} b^{*}\right)_{k} e^{-i k \lambda}\right) \cdot\left(\sum_{k=0}^{\infty}\left(C_{G} b^{*}\right)_{k} e^{-i k \lambda}\right)^{*} \vec{\gamma}^{*}, \\
& F^{0}(\lambda)+G^{0}(\lambda)=\vec{\delta}\left(\sum_{k=0}^{\infty}\left(C_{F} b^{*}\right)_{k} e^{-i k \lambda}\right) \cdot\left(\sum_{k=0}^{\infty}\left(C_{F} b^{*}\right)_{k} e^{-i k \lambda}\right)^{*} \vec{\delta}^{*} .
\end{aligned}
$$

Taking into account

$$
\frac{1}{2 \pi} \int_{-\pi}^{\pi} F(\lambda) d \lambda=P_{1}, \quad \frac{1}{2 \pi} \int_{-\pi}^{\pi} G(\lambda) d \lambda=P_{2},
$$

the unknown vectors $\vec{\beta}=\left(\beta_{1}, \ldots, \beta_{T}\right)^{\top}, \vec{\delta}=\left(\delta_{1}, \ldots, \delta_{T}\right)^{\top}$, and $b=\{b(k): k=0,1, \ldots\}$ are evaluated with the help of the canonical factorizations (3)-(5) for the densities $F^{0}(\lambda)$, $G^{0}(\lambda)$, and $F^{0}(\lambda)+G^{0}(\lambda)$.

If at least one of these spectral densities is known, one can use one of the relations (17), (18) to calculate the least favorable density. If the density $G(\lambda)$ is known, then the least favorable density $F^{0}(\lambda) \in D_{0}$ is given by

$$
F^{0}(\lambda)=\max \left\{\vec{\gamma}\left(\sum_{k=0}^{\infty}\left(\mathbf{C}_{G} b^{*}\right)_{k} e^{-i k \lambda}\right)\left(\sum_{k=0}^{\infty}\left(\mathbf{C}_{G} b^{*}\right)_{k} e^{-i k \lambda}\right)^{*} \vec{\gamma}^{*}-G(\lambda), 0\right\},
$$

where $\max \{B(\lambda), 0\}=B(\lambda)$ in the case of $B(\lambda) \geq 0$; otherwise $\max \{B(\lambda), 0\}$ means the zero matrix. If the density $F(\lambda)$ is known, then the least favorable density $G^{0}(\lambda) \in D_{0}$ is given by

$$
G^{0}(\lambda)=\max \left\{\vec{\delta}\left(\sum_{k=0}^{\infty}\left(\mathbf{C}_{F} b^{*}\right)_{k} e^{-i k \lambda}\right) \cdot\left(\sum_{k=0}^{\infty}\left(\mathbf{C}_{F} b^{*}\right)_{k} e^{-i k \lambda}\right)^{*} \vec{\delta}^{*}-F(\lambda), 0\right\} .
$$

The unknown vectors $\vec{\gamma}, \vec{\delta}$, and $b(k)$ are found from the factorization (3)-(5) of the densities $F^{0}(\lambda), G(\lambda)$, and $F^{0}(\lambda)+G(\lambda)$ (or, that of $F(\lambda), G^{0}(\lambda)$, and $F(\lambda)+G^{0}(\lambda)$ ) by using condition (19).

Therefore the following result holds.

Theorem 4.1. The least favorable in the class $D_{0,0}$ spectral densities $F^{0}(\lambda)$ and $G^{0}(\lambda)$ for the optimal filtration of the functional $A \vec{\xi}$ are given by relations (17), (18), (3)-(5), (12), (13), and (19). If the density $G(\lambda)$ (or $F(\lambda)$ ) is known and admits the canonical factorization, then the least favorable density $F^{0}(\lambda)$ (or $G^{0}(\lambda)$ ) is determined by relations (20), (3)-(5), (14), and (19) (or (21), (3)-(5), (15), and (19)). The minimax spectral characteristic of the optimal estimator $A \vec{\xi}$ is calculated by relations (8), (10).

Example 2. Consider the problem of estimation of the random variable

$$
A_{1} \xi=\xi(0)+\xi(-1)
$$

in the case where $T=1, G(\lambda)=1$, and $P_{1}=2$. The least favorable spectral density in the class $D_{0,0}$ is

$$
F^{0}(\lambda)=\max \left\{C\left|b(0)+b(1)+b(0) e^{-i \lambda}\right|^{2}-1,0\right\},
$$

where the unknown coefficients $b(0)$ and $b(1)$ form a solution of the following conditional extremum problem:

$$
\left[\begin{array}{l}
(b(0)+b(1))^{2}+b^{2}(0) \rightarrow \min , \\
b^{2}(0)+b^{2}(1)=2 b^{4}(0) .
\end{array}\right.
$$


Applying the Lagrange method to solve this problem we obtain

$$
F^{0}(\lambda)=\max \left\{C\left|\sqrt{10} / 8+\sqrt{10} / 4 e^{-i \lambda}\right|^{2}-1,0\right\},
$$

where the unknown constant $C$ is determined by the condition $(2 \pi)^{-1} \int_{-\pi}^{\pi} F^{0}(\lambda) d \lambda=1$.

Example 3. Let $G(\lambda)=\left|1+e^{-i \lambda}\right|^{2}$ in the latter example. Assume one wants to estimate $\xi(0)$. Then we get the following extremal problem:

$$
\left[\begin{array}{l}
(2 b(0)+b(1))^{2}+b^{2}(0) \rightarrow \min \\
b^{2}(0)+b^{2}(1)=3
\end{array}\right.
$$

The least favorable spectral density is given by

$$
F^{0}(\lambda)=\max \left\{C\left|\sqrt{2 / 3}+\sqrt{2 / 3} e^{-i \lambda}\right|^{2}-G(\lambda), 0\right\} .
$$

Example 4. Let $T=2, G(\lambda)=E$, and $A_{1} \vec{\xi}=\vec{a}(0) \vec{\xi}(0)+\vec{a}(1) \vec{\xi}(-1)$. The least favorable in the class $D_{0,0}$ density for the optimal extrapolation of the functional $A_{1} \vec{\xi}$ is given by equality (20), where the sequence of matrices $b=\{b(0), b(1)\}$ is a solution of the following conditional extremum problem:

$$
\left[\begin{array}{l}
(\vec{a}(0) b(0)+\vec{a}(1) b(1))(\vec{a}(0) b(0)+\vec{a}(1) b(1))^{*}+\vec{a}(1) b(0) b^{*}(0) \vec{a}^{*}(1) \rightarrow \text { max } \\
E+b(1) b^{-1}(0)\left(b^{-1}(0)\right)^{*} b^{*}(1)=b(0) P b^{*}(0), \quad P=P_{1}+P_{2} .
\end{array}\right.
$$

Consider the real case. Let $\vec{a}(0)=\vec{a}(1)=(1,1)$. Then the least favorable density in the class $D_{0,0}$ is

$$
F^{0}(\lambda)=\max \left\{\vec{\gamma}\left(d(0)+d(1) e^{-i \lambda}\right)\left(d(0)+d(1) e^{-i \lambda}\right)^{*} \vec{\gamma}^{*}-G(\lambda), 0\right\},
$$

where $d(0)=\vec{a}(0) b(0)+\vec{a}(1) b(1), d(1)=\vec{a}(1) b(0)$, and the unknown sequence of matrices $b$ is determined by the relations

$$
\left[\begin{array}{l}
b(0)+b(1)=\alpha b(1) b^{-1}(0)\left(b^{-1}(0)\right)^{*} \\
2 b(0)+b(1)=-b(0) P-\alpha b(1) b^{-1}(0)\left(b^{-1}(0)\right)^{*}\left(b^{-1}(0)\right)^{*} b^{*}(1) .
\end{array}\right.
$$

Here $\alpha$ is the Lagrange multiplier. The vector $\vec{\gamma}$ is determined from the normalizing condition $(2 \pi)^{-1} \int_{-\pi}^{\pi} F(\lambda) d \lambda=P_{1}$.

\section{Concluding Remarks}

The results of the second section allow one to find the spectral characteristic and mean square error of the optimal linear estimator of the functional

$$
A \vec{\xi}=\sum_{j=0}^{\infty} \vec{a}(j) \vec{\xi}(-j)
$$

depending on unknown values of a vector stationary sequence $\vec{\xi}(n)$. The estimator is constructed from observations of the sequence $\vec{\xi}(j)+\vec{\eta}(j)$ for $j \leq 0$, where $\vec{\eta}(n)$ is a vector stationary sequence, uncorrelated with $\vec{\xi}(n)$. The explicit optimal estimators are found for particular cases. The problem of estimation is solved in the last three sections under the condition that the densities are unknown but a class $D$ containing these spectral densities is known. The least favorable spectral densities and minimax (robust) spectral characteristics of the optimal estimators are found for some particular classes $D$. 


\section{BIBLIOGRAPHY}

1. K. S. Vastola and H. V. Poor, An analysis of the effects of spectral uncertainty on Wiener filtering, Automatica 28 (1983), 289-293.

2. S. A. Kassam and H. V. Poor, Robust techniques for signal processing: A survey, Proc. IEEE 73 (1985), no. 3, 433-481.

3. U. Grenander, A prediction problem in game theory, Ark. Mat. 3 (1957), 371-379. MR0090486 $(19: 822 \mathrm{~g})$

4. J. Franke, On the robust prediction and interpolation of time series in the presence of correlated noise, J. Time Series Analysis 5 (1984), no. 4, 227-244. MR782077 (86i:62192)

5. J. Franke, Minimax robust prediction of discrete time series, Z. Wahr. Verw. Geb. 68 (1985), 337-364. MR771471 (86f:62164)

6. M. P. Moklyachuk, Estimates of stochastic processes from observations with noise, Theory Stoch. Process. 3(19) (1997), no. 3-4, 330-338.

7. M. P. Moklyachuk, Robust procedures in time series analysis, Theory Stoch. Process. 6(22) (2000), no. 3-4, 127-147.

8. M. P. Moklyachuk, Game theory and convex optimization methods in robust estimation problems, Theory Stoch. Process. 7(23) (2001), no. 1-2, 253-264.

9. Yu. A. Rozanov, Stationary Random Processes, Second edition, "Nauka", Moscow, 1990; English transl. of the first edition, Holden-Day, San Francisco, 1967. MR1090826 (92d:60046)

Department of Probability Theory and Mathematical Statistics, Faculty for Mechanics and Mathematics, National Taras Shevchenko University, Academician Glushkov Avenue, 6 , KYIV 03127, UKRAINE

E-mail address: mmp@univ.kiev.ua

Department of Probability Theory and Mathematical Statistics, Faculty for Mechanics and Mathematics, National Taras Shevchenko University, Academician Glushkov Avenue, 6, KYIV 03127, UKRAINE

Received 20/JAN/2006

Translated by V. V. SEMENOV 